\title{
Cloaking via change of variables in electric impedance tomography
}

\author{
R V Kohn ${ }^{1}$, H Shen ${ }^{1}$, M S Vogelius ${ }^{2}$ and M I Weinstein ${ }^{3}$ \\ ${ }^{1}$ Courant Institute of Mathematical Sciences, New York University, NY, USA \\ 2 Department of Mathematics, Rutgers University, NJ, USA \\ ${ }^{3}$ Department of Applied Physics and Applied Mathematics, Columbia University, USA \\ E-mail: kohn@cims.nyu.edu, haiping@cims.nyu.edu, vogelius@math.rutgers.edu and \\ miw2103@columbia.edu
}

Received 3 August 2007, in final form 4 December 2007

Published 16 January 2008

Online at stacks.iop.org/IP/24/015016

\begin{abstract}
A recent paper by Pendry et al (2006 Science 312 1780-2) used the coordinate invariance of Maxwell's equations to show how a region of space can be 'cloaked' - in other words, made inaccessible to electromagnetic sensingby surrounding it with a suitable (anisotropic and heterogenous) dielectric shield. Essentially the same observation was made several years earlier by Greenleaf et al (2003 Math. Res. Lett. 10 685-93, 2003 Physiol. Meas. 24 413-9) in the closely related setting of electric impedance tomography. These papers, though brilliant, have two shortcomings: (a) the cloaks they consider are rather singular; and (b) the analysis by Greenleaf, Lassas and Uhlmann does not apply in space dimension $n=2$. The present paper provides a fresh treatment that remedies these shortcomings in the context of electric impedance tomography. In particular, we show how a regular near-cloak can be obtained using a nonsingular change of variables, and we prove that the change-of-variable-based scheme achieves perfect cloaking in any dimension $n \geqslant 2$.
\end{abstract}

\section{Introduction}

We say a region of space is 'cloaked' with respect to electromagnetic sensing if its contentsand even the existence of the cloak-are inaccessible to such measurements.

Is cloaking possible? The answer is yes, at least in principle. A cloaking scheme based on change-of-variables was discussed for electric impedance tomography by Greenleaf et al $[19,20]$, and for the time-harmonic Maxwell's equation by Pendry, Schurig and Smith [35, 38]. Other schemes have also been discussed, including one based on optical conformal mapping $[28,29]$, another based on anomalous localized resonances [32] and a third based on the use of sensors and active sources [30,36]. Recent developments include numerical $[6,12,46]$ and 
experimental [39] implementations of change-of-variable-based cloaking; adaptations of the change-of-variable-based scheme to acoustic or elastic sensing [13, 31]; and the introduction of related schemes for cloaking active objects such as light sources [17].

Is cloaking interesting? The answer is clearly yes. One reason is theoretical: the existence of cloaks reveals intrinsic limitations of electromagnetic-based schemes for remote sensing, such as inverse scattering and impedance tomography. A second reason is practical: cloaking provides an easy method for making any object invisible- by simply surrounding it with a cloak. The appeal of this idea has attracted a lot of attention, e.g. [8, 45].

Is cloaking practical? The answer is not yet clear. All approaches to cloaking require the design of materials with exotic dielectric properties. One hopes that the desired properties can be achieved (or at least approximated) by means of 'metamaterials' [40]; for the schemes based on change-of-variables this seems to be the case [39]. For a cloaking scheme to be practical it must be reasonably insensitive to imperfection; the robustness of the change-of-variable-based scheme has just begun to be addressed [10, 18, 37] (see section 2.3 for comments on this work.)

The present paper is related to the first and last of the preceding questions. We ask:

(i) Does the change-of-variable-based scheme really achieve a perfect cloak?

(ii) What about a regularized version of this scheme? How close does it come to achieving cloaking?

Our analysis is restricted to electric impedance tomography. This amounts to considering electromagnetic sensing in the low-frequency limit [26]; it is simpler than the finite-frequency setting, due to the ready availability of variational principles. But we do discuss the finitefrequency setting, in section 2.5 .

Concerning (i): there is a cause for concern, because the underlying change of variables is highly singular (see section 2.3). Singularities are sometimes significant; for example, the fundamental solution of Laplace's equation is harmonic except at a point. The physics literature recognizes this issue; for example, Cummer et al write in [12] that 'whether perfect cloaking is achievable, even in theory, is ... an open question'. They also suggest, using an argument based on geometrical optics, that the presence of a singularity 'may degrade cloaking performance to an unknown degree'.

Actually, (i) was settled for electric impedance tomography by [19] in space dimension $n \geqslant 3$, using a method that does not work in space dimension two. One goal of the present paper is to show that the situation is not significantly different when $n=2$ : perfect cloaking is also possible in space dimension two. Our discussion of perfect cloaking, presented in section 4, is not fundamentally different from that in [19]; in particular, our main tool (like [19]) is a result about the removability of singularities for harmonic functions. However our discussion differs from [19] by treating all dimensions $n \geqslant 2$ simultaneously, and by working directly with the divergence-form PDE of electrostatics rather than rewriting it as the LaplaceBeltrami equation of an associated Riemannian metric. In addition, our exposition is perhaps more elementary (thus more accessible to non-expert readers).

Concerning (ii): the question is as important as the answer. We suggest that the 'perfect cloak', obtained using a singular change of variables, not be taken literally. Instead, it should be used to design a more regular 'near-cloak', based on a less singular change of variable. The near-cloak is physically more plausible (for example, its dielectric tensor is strictly positive and finite). Moreover, the mathematical analysis of the near-cloak is actually easier, since nothing is singular. Basically, the problem reduces to understanding how boundary measurements are influenced by dielectric inclusions (see section 2.3 for further explanation).

The paper is organized as follows. We begin, in section 2, by introducing electric impedance tomography and giving a brief, nontechnical explanation of the change-of-variable- 
based cloaking scheme. That section also puts our work in context, discussing its relation to known uniqueness results and explaining why the finite-frequency case is similar to but different from that considered here. Then, in sections 3 and 4, we give a rigorous analysis of the change-of-variable-based cloaking scheme. In section 3 , we use a regular change of variables and prove that the inclusion is almost cloaked. In section 4 , we use a singular change of variables and prove that the inclusion is perfectly cloaked.

\section{The main ideas}

\subsection{Electric impedance tomography}

In electric impedance tomography, one uses static voltage and current measurements at the boundary of an object to gain information about its internal structure.

Mathematically, we suppose the object occupies a (known) bounded region $\Omega \subset \mathbb{R}^{n}$, $n \geqslant 2$. Its (unknown) electrical conductivity $\sigma(x)$ is a non-negative symmetric-matrix-valued function on $\Omega$. The PDE of electrostatics is

$$
\nabla \cdot(\sigma \nabla u)=\sum_{i, j} \frac{\partial}{\partial x_{i}}\left(\sigma_{i j}(x) \frac{\partial u}{\partial x_{j}}\right)=0 \quad \text { in } \Omega ; .
$$

It relates the voltage $u$ and the associated electric field $\nabla u$ to the resulting current $\sigma \nabla u$ (see section 2.5). The PDE (1) determines a 'Dirichlet-to-Neumann map' $\Lambda_{\sigma}$; by definition, it takes an arbitrary boundary voltage to the associated current flux

$$
\Lambda_{\sigma}:\left.\left.u\right|_{\partial \Omega} \rightarrow(\sigma \nabla u) \cdot v\right|_{\partial \Omega}
$$

where $v$ is the outward unit normal to $\partial \Omega$. Electric impedance tomography seeks information on $\sigma$, given knowledge of the mapping $\Lambda_{\sigma}$. In the mathematics literature this problem was first proposed and partially addressed by Calderón [7].

Does $\Lambda_{\sigma}$ determine $\sigma$ ? In general, the answer is no: the PDE is invariant under change of variables, so $\sigma$ can at best be determined 'up to change of variables'. We shall explain this statement in section 2.2. If, however, $\sigma$ is scalar-valued, positive and finite ${ }^{4}$, then the answer is basically yes: under some modest (apparently technical) conditions on the regularity of $\sigma$, knowledge of the Dirichlet-to-Neumann map $\Lambda_{\sigma}$ determines an internal isotropic conductivity $\sigma(x)$ uniquely. We shall review these results in section 2.4.

What does it mean in this context for a subset $D$ of $\Omega$ to be cloaked? In principle, it means that the contents of $D$-and even the existence of the cloak-are invisible to electrostatic boundary measurements. To keep things simple, however, we shall use a slightly more restrictive definition: we say $D \subset \Omega$ is cloaked by a conductivity distribution $\sigma_{c}(x)$ defined outside $D$ if the associated boundary measurements at $\partial \Omega$ are identical to those of a homogeneous, isotropic region with conductivity 1 -regardless of the conductivity in $D$ (see figure 1). More precisely:

Definition 1. Let $D \subset \Omega$ be fixed, and let $\sigma_{c}: \Omega \backslash D$ be a non-negative, matrix-valued conductivity defined on the complement of $D$. We say $\sigma_{c}$ cloaks the region $D$ if its extensions across $D$,

$$
\sigma_{A}(x)= \begin{cases}A(x) & \text { for } x \in D \\ \sigma_{c}(x) & \text { for } x \in \Omega \backslash D\end{cases}
$$

4 When we say $\sigma$ is 'positive and finite' we mean it is a bounded, measurable function with $\sigma(x) \geqslant c_{0}$ a.e. in $\Omega$ for some $c_{0}>0$. 


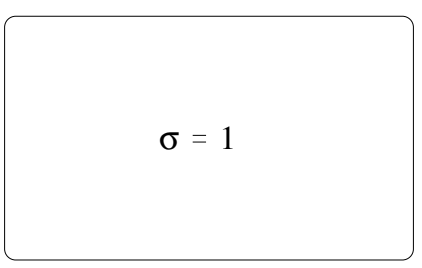

voltage f implies current flux $g$

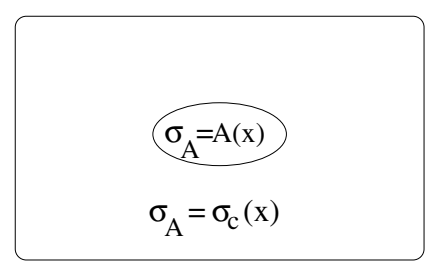

voltage f implies same current flux $\mathrm{g}$

Figure 1. The region $D$ is cloaked by $\sigma_{c}$ if, regardless of the conductivity distribution $A(x)$ in $D$, the boundary measurements at $\partial \Omega$ are identical to those of a uniform region with conductivity 1 .

produce the same boundary measurements as a uniform region with conductivity $\sigma \equiv 1$, regardless of the choice of the conductivity $A(x)$ in $D$.

The name is appropriate: a cloak makes the associated region $D$ invisible with respect to electric impedance tomography. Indeed, suppose $\sigma_{c}$ cloaks $D \subset \Omega$ in the sense of definition 1 , and let $\Omega^{\prime}$ be any domain containing $\Omega$. Then the Dirichlet-to-Neumann map of

$$
\sigma(x)= \begin{cases}A(x) & \text { for } x \in D \\ \sigma_{c}(x) & \text { for } x \in \Omega \backslash D \\ 1 & \text { for } x \in \Omega^{\prime} \backslash \Omega\end{cases}
$$

is independent of $A$, and identical to that of the domain $\Omega^{\prime}$ with constant conductivity 1 . This holds because $\Omega$ communicates with its exterior only through its Dirichlet-to-Neumann map.

Note that from a single example of cloaking, this extension argument produces many other examples. Indeed, according to (4), if $\sigma_{c}$ cloaks $D \subset \Omega$ in the sense of definition 1, then the extension of $\sigma_{c}$ by 1 cloaks $D$ in any larger domain $\Omega^{\prime}$.

We shall explain in section 2.3, following [20,35], how the invariance of electrostatics under change of variables leads to examples of cloaks.

\subsection{Invariance by change of variables}

The invariance of the PDE (1) by change of variables is well known. So is the fact that $\Lambda_{\sigma}$ can determine $\sigma$ at best 'up to change of variables'. This observation is explicit, e.g., in $[22,25]$, with an attribution to Luc Tartar.

It is convenient to think variationally. Recall that if $\sigma(x)$ is bounded and positive definite, then the solution of (1) with Dirichlet data $f$ solves the variational problem

$$
\min _{u=f \text { at } \partial \Omega} \int_{\Omega}\langle\sigma \nabla u, \nabla u\rangle \mathrm{d} x .
$$

Moreover, the minimum 'energy' is determined by $\Lambda_{\sigma}$, since when $u$ solves (1) we have

$$
\int_{\Omega}\langle\sigma \nabla u, \nabla u\rangle \mathrm{d} x=\int_{\partial \Omega} f \Lambda_{\sigma}(f) .
$$

Thus, knowledge of $\Lambda_{\sigma}$ determines the minimum energy, viewed as a quadratic form on Dirichlet data. The converse is also true: knowledge of the minimum energy for all Dirichlet data determines the boundary map $\Lambda_{\sigma}$. This follows from the well-known polarization identity: for any $f$ and $g$,

$$
4 \int_{\partial \Omega} f \Lambda_{\sigma} g=\int_{\partial \Omega}(f+g) \Lambda_{\sigma}(f+g)-\int_{\partial \Omega}(f-g) \Lambda_{\sigma}(f-g) .
$$


The right-hand side is the minimum energy for $f+g$ minus that for $f-g$, while the left-hand side is the boundary map, viewed as a bilinear form on Dirichlet data.

We turn now to change of variables. Suppose $y=F(x)$ is an invertible, orientationpreserving change of variables on $\Omega$. Then we can change variables in the variational principle (5):

$$
\int_{\Omega} \sum \sigma_{i j} \frac{\partial u}{\partial x_{i}} \frac{\partial u}{\partial x_{j}} \mathrm{~d} x=\int_{\Omega} \sum \sigma_{i j} \frac{\partial u}{\partial y_{k}} \frac{\partial y_{k}}{\partial x_{i}} \frac{\partial u}{\partial y_{l}} \frac{\partial y_{l}}{\partial x_{j}} \operatorname{det}\left(\frac{\partial x}{\partial y}\right) \mathrm{d} y .
$$

We can write this more compactly as

$$
\int_{\Omega}\left\langle\sigma(x) \nabla_{x} u, \nabla_{x} u\right\rangle \mathrm{d} x=\int_{\Omega}\left\langle F_{*} \sigma(y) \nabla_{y} u, \nabla_{y} u\right\rangle \mathrm{d} y
$$

where

$$
F_{*} \sigma(y)=\frac{1}{\operatorname{det}(D F)(x)} D F(x) \sigma(x)(D F(x))^{T}
$$

in which $D F$ is the matrix with $i, j$ element $\partial y_{i} / \partial x_{j}$ and the right-hand side is evaluated at $x=F^{-1}(y)$. We call $F_{*} \sigma$ the push-forward of $\sigma$ by the change of variables $F$.

We come finally to the main point: if $F(x)=x$ at $\partial \Omega$, then the boundary measurements associated with $\sigma$ and $F_{*} \sigma$ are identical; in other words,

$$
\Lambda_{\sigma}(f)=\Lambda_{F_{*} \sigma}(f) \quad \text { for all } f \text {. }
$$

Indeed, if $F(x)=x$ at $\partial \Omega$ then the change of variables does not affect the Dirichlet data. So for any $f$,

$$
\begin{aligned}
\int_{\partial \Omega} f \Lambda_{\sigma} f & =\min _{u=f \text { at } \partial \Omega} \int_{\Omega}\left\langle\sigma(x) \nabla_{x} u, \nabla_{x} u\right\rangle \mathrm{d} x \\
& =\min _{u=f \text { at } \partial \Omega} \int_{\Omega}\left\langle F_{*} \sigma(y) \nabla_{y} u, \nabla_{y} u\right\rangle \mathrm{d} y \\
& =\int_{\partial \Omega} f \Lambda_{F_{*} \sigma} f .
\end{aligned}
$$

Thus $\Lambda_{\sigma}$ and $\Lambda_{F_{*} \sigma}$ determine identical quadratic forms, from which it follows by (7) that $\Lambda_{\sigma}=\Lambda_{F_{*} \sigma}$.

\subsection{Cloaking via change of variables}

We now explain how change-of-variable-based cloaking works. For simplicity we focus on the radial case: $\Omega=B_{2}$ is a ball of radius 2 , and the region $D$ to be cloaked is $B_{1}$, the concentric ball of radius 1 (see figure 2). It will be clear, however, that the method is much more general.

We start by explaining how $B_{1}$ can be nearly cloaked using a regular change of variables. Fixing a small parameter $\rho>0$, consider the piecewise-smooth change of variables

$$
F(x)= \begin{cases}\frac{x}{\rho} & \text { if } \quad|x| \leqslant \rho \\ \left(\frac{2-2 \rho}{2-\rho}+\frac{1}{2-\rho}|x|\right) \frac{x}{|x|} & \text { if } \quad \rho \leqslant|x| \leqslant 2 .\end{cases}
$$

Its key properties are that

- $F$ is continuous and piecewise smooth;

- $F$ expands $B_{\rho}$ to $B_{1}$, while mapping the full domain $B_{2}$ to itself;

- $F(x)=x$ at the outer boundary $|x|=2$. 


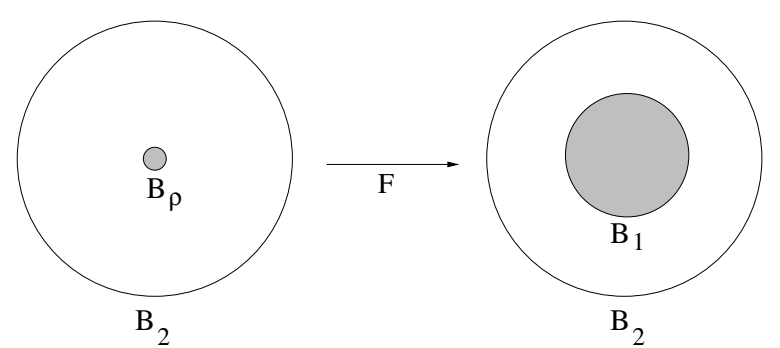

Figure 2. The change of variables leading to a regular near-cloak: $F$ expands a small ball $B_{\rho}$ to a ball of radius 1 .

The associated near-cloak is the push-forward via $F$ of the constant conductivity $\sigma=1$, restricted to the annulus $B_{2} \backslash B_{1}$. (Abusing notation a bit, we write this as $F_{*} 1$.) To explain why, consider any conductivity of the form

$$
\sigma_{A}(y)= \begin{cases}A(y) & \text { for } y \in B_{1} \\ F_{*} 1(y) & \text { for } y \in B_{2} \backslash B_{1} .\end{cases}
$$

By the change-of-variables principle (9), its boundary measurements are identical to those of

$$
F_{*}^{-1} \sigma_{A}(x)= \begin{cases}F_{*}^{-1} A(x) & \text { for } x \in B_{\rho} \\ 1 & \text { for } x \in B_{2} \backslash B_{\rho}\end{cases}
$$

where

$$
F_{*}^{-1} \sigma_{A}=\left(F^{-1}\right)_{*} \sigma_{A}
$$

denotes the push-forward of the conductivity distribution $\sigma_{A}$ by the map $F^{-1}$. Thus, the boundary measurements associated with $\sigma_{A}$ are the same as those of a uniform ball perturbed by a small inclusion at the centre. The contents of the inclusion are uncontrolled, since $A$ is arbitrary. But the radius of the inclusion is small, namely $\rho$. As we explain in section 3 , this is enough to assure that the boundary measurements are close to those of a completely uniform ball. Thus when $\rho$ is sufficiently small, this scheme comes close to cloaking the unit ball (see theorem 1 in section 3.3).

Now we show how $B_{1}$ can be perfectly cloaked using a singular change of variables. The idea is obvious: just take $\rho=0$ in (10). The resulting change of variables

$$
F(x)=\left(1+\frac{1}{2}|x|\right) \frac{x}{|x|}
$$

is the same one used in [19, 20] for electrostatics and in [35] for electromagnetics. Its key properties are that

- $F$ is smooth except at 0 ;

- $F$ blows up the point 0 to the ball $B_{1}$, while mapping the full domain $B_{2}$ to itself;

- $F(x)=x$ at the outer boundary $|x|=2$.

A heuristic 'proof' that $F_{*} 1$ gives a perfect cloak uses the same argument as before. This time $F_{*}^{-1} A$ occupies a point rather than a ball. Changing the conductivity at a point should have no effect on the boundary measurements. Therefore we expect that when $\sigma_{A}$ is given by (11) with $F$ given by (12), the boundary measurements should be identical to those obtained for a uniform ball with $\sigma \equiv 1$.

This heuristic proof needs some clarification. The validity of the change-of-variables formula is open to question when $F$ is so singular. Worse: our cloak $F_{*} 1$ is quite singular 
near its inner boundary $|x|=1$; some care is therefore needed concerning what we mean by a solution of the PDE (1). These topics will be addressed in section 4.

We have focused on the radial case because the simple, explicit form of the diffeomorphism $F$ leads to an equally simple, explicit formula for the associated cloak (see section 4.1). However, the method is clearly not limited to the radial case (see theorems 2 and 4).

Our 'regular near-cloak' is quite different from the approximate cloaking scheme considered in $[18,37]$. Those papers start with a perfect cylindrical cloak, obtained using the $2 \mathrm{D}$ version of the familiar construction (12). This cloak fills the annulus $1<|y|<2$ with an anisotropic, heterogeneous medium, whose behaviour is rather singular near the inner boundary $|y|=1$ (see section 4.1 ). The approximate cloak considered in $[18,37]$ is obtained by restricting the perfect cloak to a slightly smaller annulus $1+\delta<|y|<2$. Perfect cloaking (at any frequency) is obtained as $\delta \rightarrow 0$; however the convergence is extremely slow. The convergence can be greatly improved by introducing a layer at the edge of the cloak that permits surface currents [18]. In summary: our 'regular near-cloak' avoids singular behaviour by using a regularized change of variables, whereas [18, 37] avoid singular behaviour by truncation. We also note the interesting paper [10], which explores the sensitivity of the ideal cloak to various types of material or manufacturing imperfections.

The focus of this paper is on cloaking. But we note in passing that it might be possible to design other interesting devices using similar change-of-variable-based techniques. A recent example of this type is the scheme of [9] for rotating electromagnetic fields.

\subsection{Relation to known uniqueness results}

The uniqueness problem for electric impedance tomography asks whether it is possible, in principle, to determine $\sigma(x)$ using boundary measurements. In other words, does $\Lambda_{\sigma}$ determine $\sigma$ ?

If it is known in advance that the conductivity is scalar-valued, positive and finite, then the answer is basically yes. The earliest uniqueness results-in the class of analytic or piecewise analytic conductivities-date from the early 1980s [14, 23, 24]. A few years later, using entirely different methods, uniqueness was proved for conductivities that are several times differentiable in dimensions $n \geqslant 3$ [42] and in dimension $n=2$ [33]. Recently, using yet another method, uniqueness has been shown in two space dimensions with no regularity hypothesis at all, assuming only that $\sigma(x)$ is scalar-valued, strictly positive and finite [4]. We have given just a few of the most important references; for more complete surveys, see $[11,21,43,44]$.

We observed in section 2.2 that when $\sigma(x)$ is symmetric-matrix-valued, boundary measurements can at best determine it 'up to change of variables'. Is this the only invariance? In other words, if two conductivities give the same boundary measurements, must they be related by change of variables? If cloaking is possible then the answer should be no, since the conductivities $\sigma_{A}$ in (3) are not related, as $A$ varies, by change of variables.

Paradoxically, Sylvester proved that in two space dimensions, boundary measurements do determine $\sigma$ up to change of variables [41]! 5 The heart of his proof was the introduction of isothermal coordinates-i.e. construction of a (unique) map $G: \Omega \rightarrow \Omega$ such that $G_{*} \sigma$ is isotropic and $G(x)=x$ at $\partial \Omega$. By uniqueness in the isotropic setting, $\Lambda_{\sigma}$ determines $G_{*} \sigma$; thus boundary measurements determine $\sigma$ up to change of variables.

Does cloaking contradict Sylvester's result? Not at all. The resolution of the paradox is that the introduction of isothermal coordinates depends crucially on having upper and

5 Sylvester's paper proved only a local result, and required $\sigma$ to be $C^{3}$. When combined with [33], however, his analysis gives a global $C^{3}$ result. The recent improvement in [5] assumes only that $\sigma$ is bounded and positive-definite. 
lower bounds for $\sigma(x)$. Indeed, if $\Omega$ is a ball and $\sigma=F_{*} 1$ with $F$ given by (10), then the associated isothermal coordinate transformation is $G=F^{-1}$. As $\rho \rightarrow 0$ in (10) the isothermal coordinates become singular. When $\rho$ is positive we do not get perfect cloaking (consistent with the Sylvester's theorem). When $\rho=0$ we do get cloaking-but the eigenvalues of $\sigma$ are unbounded both above and below near $|x|=1$ (see section 4.1), Sylvester's argument no longer applies, and indeed there is no isothermal coordinate system.

Do boundary measurements determine $\sigma$ up to change of variables in three or more space dimensions? If we assume only that $\sigma$ is non-negative then the answer is no, since cloaking is possible. If, however, we assume that $\sigma$ is strictly positive and finite, then such a result could still be true. A proof for real-analytic conductivities is given in [27].

\subsection{Comments on cloaking at nonzero frequency}

This paper focuses on electric impedance tomography because we can explain the essence of change-of-variable-based cloaking in this electrostatic setting with a minimum of mathematical complexity. The practical applications of cloaking are, however, mainly at nonzero frequencies - for example, making objects invisible at optical wavelengths, or undetectable by electromagnetic scattering measurements. We therefore discuss briefly how the positivefrequency problem is similar to, yet different from, the static case.

For time-harmonic fields in a linear medium, Maxwell's equations become

$$
\nabla \times H=(\sigma-\mathrm{i} \omega \epsilon) E, \quad \nabla \times E=\mathrm{i} \omega \mu H .
$$

Here $E$ and $H$ are complex vector fields representing the electric and magnetic fields; $\sigma, \epsilon$ and $\mu$ are real-valued, positive-definite symmetric tensors representing the electrical conductivity, dielectric permittivity and magnetic permeability of the medium; and $\omega>0$ is the frequency. The physical electric and magnetic fields are $\operatorname{Re}\left\{E \mathrm{e}^{-\mathrm{i} \omega t}\right\}$ and $\operatorname{Re}\left\{H \mathrm{e}^{-\mathrm{i} \omega t}\right\}$.

When $\omega=0$, (13) reduces formally to (1). Indeed, Maxwell's equations become $\nabla \times H=\sigma E$ and $\nabla \times E=0$. The latter implies $E=\nabla u$ and the former implies that $\sigma \nabla u$ is divergence-free.

The analogue of the Dirichlet-to-Neumann map $\Lambda_{\sigma}$ at finite frequency is the correspondence between the tangential component of $E$ and the tangential component of $H$ at $\partial \Omega$. When $\omega$ is not an eigenfrequency this can be expressed as a map from $\left.E\right|_{\partial \Omega} \times v$ to $\left.H\right|_{\partial \Omega} \times v$, sometimes known as the admittance. (When $\omega$ is an eigenfrequency the map is not well defined and one should consider instead all pairs $\left(\left.E\right|_{\partial \Omega} \times v,\left.H\right|_{\partial \Omega} \times v\right)$.) Mathematically, the admittance specifies the set of possible Cauchy data for (13) at frequency $\omega$. Physically, a body interacts with its exterior only through its admittance; therefore, two objects with the same admittance are indistinguishable by electromagnetic measurements at frequency $\omega$-for example, by scattering measurements.

Digressing a bit, we remark that many of the uniqueness results sketched in section 2.4 have been extended to finite frequency. In particular, the admittance of a 3D body at a single frequency determines $\sigma, \mu$ and $\epsilon$ provided they are known in advance to be scalar-valued, sufficiently smooth and constant near the boundary [34]. A different connection between the positive-frequency and electrostatic cases is provided by [26], which shows that the admittance determines the electrostatic Dirichlet-to-Neumann map in the limit $\omega \rightarrow 0$.

Let us focus now on cloaking. The positive-frequency analogue of our definition of cloaking is clear: three non-negative matrix-valued functions $\sigma, \epsilon$ and $\mu$ defined on $\Omega \backslash D$ cloak a region $D$ if the associated admittance at $\partial \Omega$ does not depend on how $\sigma, \epsilon$ and $\mu$ are extended across $D$. The positive-frequency analogue of our change-of-variables scheme is also clear: if $\Omega=B_{2}, D=B_{1}$ and $F(x)=\left(1+\frac{1}{2}|x|\right) \frac{x}{|x|}$ as in (12) and [19, 20, 35], we 
should be able to cloak $D$ by taking $\left.\sigma\right|_{\Omega \backslash D},\left.\epsilon\right|_{\Omega \backslash D}$ and $\left.\mu\right|_{\Omega \backslash D}$ each to be the 'push-forward' of the constant 1. The correctness of this scheme is demonstrated in [17] though it is not the main focus of that paper. Their argument is, roughly speaking, a finite-frequency (and more general) analogue of the one presented here in section 4 .

What about our regular near-cloak? The discussion in section 3 has an obvious extension to the time-harmonic Maxwell setting. To analyse the performance of this near-cloak, we would need an estimate for the effect of a small inclusion (with uncontrolled dielectric properties) upon the boundary measurements (admittance). Unfortunately, this question is to the best of our knowledge open, though the effect of a uniform inclusion is very well understood [3]. We anticipate a result similar to the electrostatic setting - the effect of an inclusion should tend to zero as its radius tends to zero. Such a result would, as an immediate consequence, extend the analysis of section 3 to the time-harmonic Maxwell setting.

We refer to [17] for further discussion of the time-harmonic problem. That paper includes, among other things, a new change-of-variable-based scheme for cloaking an active device (such as a light source).

\section{Analysis of the regular near-cloak}

This section reviews some well-known facts about the Dirichlet-to-Neumann map, then analyses the near-cloak obtained using the change of variable (10).

\subsection{The Dirichlet-to-Neumann map}

In discussing the PDE (1), we assume throughout this section that the conductivity is strictly positive and bounded in the sense that for some constants $0<m, M<\infty$,

$$
m|\xi|^{2} \leqslant\langle\sigma(x) \xi, \xi\rangle \leqslant M|\xi|^{2}
$$

for all $x \in \Omega$ and $\xi \in \mathbb{R}^{n}$. Our discussion of cloaking focused on the case when $\Omega$ is a ball, but in this section $\Omega$ can be any bounded domain in $\mathbb{R}^{n}$ with sufficiently regular boundary.

We will make essential use of the variational principle (5). Therefore, we must restrict our attention to Dirichlet data $f$ for which there exists a 'finite energy' solution. When $\sigma$ satisfies (14) it is well known that this occurs precisely when

$$
f \in H^{1 / 2}(\partial \Omega)=\left\{f: f=\left.v\right|_{\partial \Omega} \text { for some } v \text { such that } \int_{\Omega}|\nabla v|^{2} \mathrm{~d} x<\infty\right\} .
$$

When $f$ is constant the solution is also constant-a trivial case-so it is natural to restrict attention to the subspace $H_{*}^{1 / 2}(\partial \Omega)=H^{1 / 2}(\partial \Omega) \cap\left\{\int_{\partial \Omega} f=0\right\}$, with the natural norm

$$
\|f\|_{H_{*}^{1 / 2}(\partial \Omega)}^{2}=\min _{v=f \text { at } \partial \Omega} \int_{\Omega}|\nabla v|^{2} \mathrm{~d} x .
$$

This is a fractional Sobolev space, consisting of functions with 'one-half derivative in $L^{2}(\partial \Omega)$ ' (see e.g. [1]). We shall not try to explain what this means in general, but we note that when $\Omega$ is a ball $B_{R}$ in $\mathbb{R}^{2}$ the interpretation is quite simple. In fact, if $f=$ $\sum_{k=1}^{\infty} a_{k} \sin (k \theta)+b_{k} \cos (k \theta)$ at the boundary then the optimal $v$ for (15) is the harmonic function $v=\sum_{k=1}^{\infty}(r / R)^{k}\left(a_{k} \sin (k \theta)+b_{k} \cos (k \theta)\right)$, and a direct calculation gives

$$
\|f\|_{H_{*}^{1 / 2}\left(\partial B_{R}\right)}^{2}=\pi \sum_{k=1}^{\infty} k\left(a_{k}^{2}+b_{k}^{2}\right) \text {. }
$$

Sometimes it is convenient to specify Neumann rather than Dirichlet data. Note that when $\sigma$ is anisotropic, the phrase 'Neumann data' refers to $g=(\sigma \nabla u) \cdot v$. It is well known that the 
space of finite energy Neumann data is $H_{*}^{-1 / 2}(\partial \Omega)=H^{-1 / 2}(\partial \Omega) \cap\left\{\int_{\partial \Omega} f=0\right\}$. It consists of mean-value-zero functions with 'minus one-half derivative in $L^{2}(\partial \Omega)$ '. In general,

$$
\|g\|_{H_{*}^{-1 / 2}(\partial \Omega)}=\sup \left\{\int_{\partial \Omega} f g:\|f\|_{H_{*}^{1 / 2}(\partial \Omega)} \leqslant 1\right\} ;
$$

when $\Omega$ is a ball of radius $R$ in $\mathbb{R}^{2}$ and $g=\sum_{k=1}^{\infty} a_{k} \sin (k \theta)+b_{k} \cos (k \theta)$ this reduces to

$$
\|g\|_{H_{*}^{-1 / 2}\left(\partial B_{R}\right)}^{2}=\pi R^{2} \sum_{k=1}^{\infty} k^{-1}\left(a_{k}^{2}+b_{k}^{2}\right) .
$$

We defined the Dirichlet-to-Neumann map $\Lambda_{\sigma}$ in (2) as the operator that takes Dirichletto-Neumann data. It is a bounded linear map from $H_{*}^{1 / 2}(\partial \Omega)$ to $H_{*}^{-1 / 2}(\partial \Omega)$. Moreover it is positive and symmetric (in the $L^{2}$ inner product) and invertible, so it defines a positive-definite quadratic form on $H_{*}^{1 / 2}(\partial \Omega)$. This form can be written 'explicitly' as

$$
\left\langle\Lambda_{\sigma} f_{1}, f_{2}\right\rangle=\int_{\partial \Omega} \Lambda_{\sigma}\left(f_{1}\right) f_{2}=\int_{\Omega}\left\langle\sigma \nabla u_{1}, \nabla u_{2}\right\rangle \mathrm{d} x
$$

where $u_{1}$ and $u_{2}$ solve the PDE (1) with Dirichlet data $f_{1}$ and $f_{2}$ respectively. The natural norm on symmetric linear maps of this type is

$$
\|\Lambda\|=\sup \left\{|\langle\Lambda f, f\rangle|:\|f\|_{H_{*}^{1 / 2}(\partial \Omega)} \leqslant 1\right\} .
$$

This is equivalent to the operator norm of $\Lambda$ viewed as a map from $H_{*}^{1 / 2}$ to $H_{*}^{-1 / 2}$, as a consequence of the polarization identity (7).

When two conductivities are ordered, the associated Dirichlet-to-Neumann maps are also ordered. More precisely, if $\sigma$ and $\eta$ satisfy

$$
\langle\sigma(x) \xi, \xi\rangle \leqslant\langle\eta(x) \xi, \xi\rangle
$$

for all $x \in \Omega$ and all $\xi \in \mathbb{R}^{n}$ then $\Lambda_{\sigma} \leqslant \Lambda_{\eta}$ in the sense that

$$
\left\langle\Lambda_{\sigma}(f), f\right\rangle \leqslant\left\langle\Lambda_{\eta}(f), f\right\rangle
$$

for all $f \in H_{*}^{1 / 2}(\partial \Omega)$. This follows easily from the variational principle (5), since if $\nabla \cdot(\sigma \nabla u)=0$ and $\nabla \cdot(\eta \nabla v)=0$ in $\Omega$ with $u=v=f$ at $\partial \Omega$, then

$$
\begin{aligned}
\left\langle\Lambda_{\sigma} f, f\right\rangle & =\int_{\Omega}\langle\sigma \nabla u, \nabla u\rangle \\
& \leqslant \int_{\Omega}\langle\sigma \nabla v, \nabla v\rangle \\
& \leqslant \int_{\Omega}\langle\eta \nabla v, \nabla v\rangle=\left\langle\Lambda_{\eta} f, f\right\rangle .
\end{aligned}
$$

\subsection{Dielectric inclusions}

The simplest special case of our PDE (1) is when $\sigma \equiv 1$. Then the solution $u$ is harmonic. We understand almost everything about harmonic functions and the associated Dirichlet-toNeumann map.

Another relatively simple case arises when $\sigma$ is uniform except for a constant-conductivity spherical inclusion of radius $\rho$ centred at some $x_{0} \in \Omega$ :

$$
\sigma_{\alpha, \rho}(x)= \begin{cases}\alpha & \text { for } x \in B_{\rho}\left(x_{0}\right) \\ 1 & \text { for } x \in \Omega \backslash B_{\rho}\left(x_{0}\right) .\end{cases}
$$


In view of (17), the effect of the inclusion depends monotonically on its conductivity $\alpha$. It is therefore natural to consider the extreme limits of the associated voltages $u_{\alpha}^{\rho}$ as $\alpha \rightarrow 0$ and $\alpha \rightarrow \infty$.

We now discuss these limits in detail, since they are important to our analysis. Given any $f \in H^{1 / 2}(\partial \Omega)$ let $u_{0}^{\rho}$ denote the solution to

$\Delta u_{0}^{\rho}=0$ in $\Omega \backslash \overline{B_{\rho}\left(x_{0}\right)}, \quad$ with $\quad \frac{\partial u_{0}^{\rho}}{\partial \nu}=0$ on $\partial B_{\rho}\left(x_{0}\right), \quad$ and $\quad u_{0}^{\rho}=f$ on $\partial \Omega$.

Similarly let $u_{\infty}^{\rho}$ denote the solution to

$\Delta u_{\infty}^{\rho}=0$ in $\Omega \backslash \overline{B_{\rho}\left(x_{0}\right)}$, with $\quad u_{\infty}^{\rho}=c_{\infty}$ on $\partial B_{\rho}\left(x_{0}\right), \quad$ and $\quad u_{\infty}^{\rho}=f$ on $\partial \Omega$,

where the constant $c_{\infty}$ is (uniquely) determined by

$$
\int_{\partial B_{\rho}\left(x_{0}\right)} \frac{\partial u_{\infty}^{\rho}}{\partial v}=0 .
$$

Using very standard energy arguments it is easy to see that

$$
u_{\alpha}^{\rho} \rightarrow u_{0}^{\rho} \quad \text { as } \quad \alpha \rightarrow 0, \quad \text { and } \quad u_{\alpha}^{\rho} \rightarrow u_{\infty}^{\rho} \quad \text { as } \quad \alpha \rightarrow \infty
$$

weakly in $H^{1}\left(\Omega \backslash \overline{B_{\rho}\left(x_{0}\right)}\right)$. Indeed, energy considerations immediately yield that $\left\|\nabla u_{\alpha}^{\rho}\right\|_{L^{2}\left(\Omega \backslash \overline{B_{\rho}\left(x_{0}\right)}\right)}$ is bounded uniformly in $\alpha$, that $\left\|\nabla u_{\alpha}^{\rho}\right\|_{L^{2}\left(B_{\rho}\left(x_{0}\right)\right)} \rightarrow 0$ as $\alpha \rightarrow \infty$, and that $\left\|\alpha \nabla u_{\alpha}^{\rho}\right\|_{L^{2}\left(B_{\rho}\left(x_{0}\right)\right)} \rightarrow 0$ as $\alpha \rightarrow 0$. By extraction of subsequences we now get weak $H^{1}\left(\Omega \backslash \overline{B_{\rho}\left(x_{0}\right)}\right)$ limits, $u_{0}^{\rho}$ and $u_{\infty}^{\rho}$, that satisfy (19) and (20), respectively. The boundary conditions on $\partial B_{\rho}\left(x_{0}\right)$ follow from the continuity of $(\sigma \nabla u) \cdot v$ and $u$ across this 'interface'. Condition (21), determining $c_{\infty}$, follows since

$$
\int_{\partial B_{\rho}\left(x_{0}\right)} \frac{\partial u_{\alpha}^{\rho+}}{\partial v}=\int_{\partial B_{\rho}\left(x_{0}\right)} \alpha \frac{\partial u_{\alpha}^{\rho-}}{\partial v}=0,
$$

and therefore

$$
\int_{\partial B_{\rho}\left(x_{0}\right)} \frac{\partial u_{\infty}^{\rho}}{\partial v}=\lim _{\alpha \rightarrow \infty} \int_{\partial B_{\rho}\left(x_{0}\right)} \frac{\partial u_{\alpha}^{\rho+}}{\partial v}=0 .
$$

It is not hard to see that this same $c_{\infty}$ may also be characterized as the constant that gives rise to the smallest energy ( of $u_{\infty}^{\rho}$ ). The fact that we get single limits as $\alpha \rightarrow 0$ and $\alpha \rightarrow \infty$, respectively, is a consequence of the uniqueness of the solution to (19) and the solution to (20). We now define

$$
\Lambda_{0}^{\rho} f=\left.\nabla u_{0}^{\rho} \cdot \nu\right|_{\partial \Omega}
$$

and

$$
\Lambda_{\infty}^{\rho} f=\left.\nabla u_{\infty}^{\rho} \cdot v\right|_{\partial \Omega}
$$

Integration by parts, together with the weak $H^{1}$ convergence, gives that $\Lambda_{\sigma_{\alpha, \rho}} f \rightarrow \Lambda_{0}^{\rho} f$ and $\Lambda_{\sigma_{\alpha, \rho}} f \rightarrow \Lambda_{\infty}^{\rho} f$ as $\alpha \rightarrow 0$ and $\alpha \rightarrow \infty$, respectively. In particular,

$$
\left\langle\Lambda_{\sigma_{\alpha, \rho}} f, f\right\rangle \rightarrow\left\langle\Lambda_{0}^{\rho} f, f\right\rangle \quad \text { as } \quad \alpha \rightarrow 0
$$

and

$$
\left\langle\Lambda_{\sigma_{\alpha, \rho}} f, f\right\rangle \rightarrow\left\langle\Lambda_{\infty}^{\rho} f, f\right\rangle \quad \text { as } \quad \alpha \rightarrow \infty
$$

Finally we note that if $\Omega$ is a ball of radius $R$ in $\mathbb{R}^{2}$ and the inclusion lies at its centre, then the above convergence of the Dirichlet-to-Neumann maps can easily be derived by explicit solution of the PDE's using separation of variables. 
In the small-particle limit $\rho \rightarrow 0$, the perturbation introduced by the presence of a small inclusion (extreme or not) is well understood. We shall not use its exact form; rather what matters to us is its magnitude, which is proportional to the volume of the inclusion.

Proposition 1. Let $\Lambda_{1}$ be the Dirichlet-to-Neumann map when $\sigma \equiv 1$, and let $\Lambda_{0}^{\rho}$ and $\Lambda_{\infty}^{\rho}$ be the Dirichlet-to-Neumann maps associated with the problems (19) and (20) respectively. Then

$$
\left\|\Lambda_{1}-\Lambda_{0}^{\rho}\right\| \leqslant C \rho^{n} \quad \text { and } \quad\left\|\Lambda_{1}-\Lambda_{\infty}^{\rho}\right\| \leqslant C \rho^{n}
$$

when $\rho$ is sufficiently small. Here, $n$ is the spatial dimension and we mean the operator norm (16) on the left-hand side of each inequality.

A proof of the estimate for $\Lambda_{1}-\Lambda_{\infty}^{\rho}$ is given in section 2 of [16] and the same argument can be used for $\Lambda_{1}-\Lambda_{0}^{\rho}$. The constant $C$ depends, of course, on the location of $x_{0}$ and the shape of $\Omega$. Much more detailed results are known, including a full asymptotic expansion for the dependence of the Dirichlet-to-Neumann map on $\rho$; see e.g. [2] for a recent review.

We have focused on spherical inclusions only for the sake of simplicity. The preceding discussion extends straightforwardly to inclusions of any fixed shape, i.e. to the situation when $B_{\rho}\left(x_{0}\right)$ is replaced by $x_{0}+\rho D$ where $D$ is any 'inclusion shape' (a bounded domain in $\mathbb{R}^{n}$, containing the origin, with sufficiently regular boundary).

\subsection{The regular near-cloak is almost invisible}

Now consider the 'regular near-cloak' discussed in section 2.3: $\Omega=B_{2}$ is a ball about the origin of radius 2 , and $\sigma=\sigma_{A}$ has the form

$$
\sigma_{A}(y)= \begin{cases}A(y) & \text { for } y \in B_{1} \\ F_{*} 1(y) & \text { for } y \in B_{2} \backslash B_{1} .\end{cases}
$$

where $F$ is given by (10). The symbol $A$ stands for 'arbitrary:' $A(x)$ is the (scalar or matrix-valued) conductivity in the region being cloaked. We assume it is positive definite and finite,

$$
m|\xi|^{2} \leqslant\langle A(y) \xi, \xi\rangle \leqslant M|\xi|^{2} \quad \text { for } \quad y \in B_{1},
$$

so the solution of the PDE (1) is well defined and unique. However our estimates will not depend on the lower and upper bounds $m$ and $M$.

As we explained in section 2.3, the Dirichlet-to-Neumann map of $\sigma_{A}$ is identical to that of

$$
F_{*}^{-1} \sigma_{A}(x)= \begin{cases}F_{*}^{-1} A(x) & \text { for } x \in B_{\rho} \\ 1 & \text { for } x \in B_{2} \backslash B_{\rho} .\end{cases}
$$

By the ordering relation (17), and the convergence results described in the previous section, we conclude that

$$
\lim _{\alpha \rightarrow 0} \Lambda_{\sigma_{\alpha, \rho}}=\Lambda_{0}^{\rho} \leqslant \Lambda_{\sigma_{A}}=\Lambda_{F_{*}^{-1} \sigma_{A}} \leqslant \Lambda_{\infty}^{\rho}=\lim _{\alpha \rightarrow \infty} \Lambda_{\sigma_{\alpha, \rho}}
$$

whence

$$
\Lambda_{0}^{\rho}-\Lambda_{1} \leqslant \Lambda_{\sigma_{A}}-\Lambda_{1} \leqslant \Lambda_{\infty}^{\rho}-\Lambda_{1}
$$

It follows using proposition 1 that the boundary measurements obtained using this near-cloak are almost identical to those of a uniform ball with conductivity 1 :

$$
\left\|\Lambda_{\sigma_{A}}-\Lambda_{1}\right\| \leqslant C \rho^{n}
$$




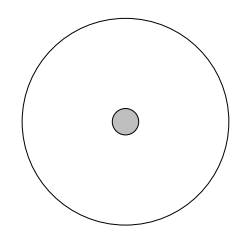

$\mathrm{G}^{-1}$

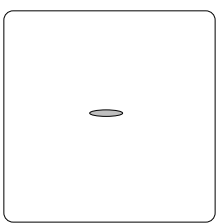

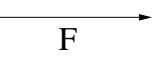

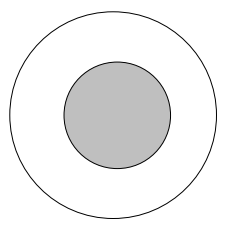

G

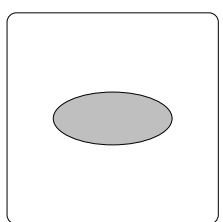

Figure 3. The map $H=G \circ F \circ G^{-1}$ blows up $G\left(B_{\rho}\right)$ to $D=G\left(B_{1}\right)$ while acting as the identity on $\partial \Omega=\partial G\left(B_{2}\right)$.

where the left-hand side is the operator norm (16). The constant $C$ is independent of $A$; in fact, it does not even depend on the values of $m$ and $M$ in (22). We have proved:

Theorem 1. Suppose the shell $B_{2} \backslash B_{1}$ has conductivity $F_{*} 1$, where $F$ is given by (10). If $\rho$ is sufficiently small then $B_{1}$ is nearly cloaked, in the sense made precise by (23).

We have focused on the spherically symmetric setting due to its simple, explicit character. However, our argument did not use this symmetry in any essential way. Indeed, the same argument proves (see figure 3):

Theorem 2. Let $G: B_{2} \rightarrow \Omega$ be a Lipschitz continuous map with a Lipschitz continuous inverse, and let $D=G\left(B_{1}\right)$. Then $H=G \circ F \circ G^{-1}: \Omega \rightarrow \Omega$ is piecewise Lipschitz; moreover

- $H$ expands $G\left(B_{\rho}\right)$ to $D$;

- $H(x)=x$ at $\partial \Omega$.

If the shell $\Omega \backslash D$ has conductivity $H_{*} 1$ then $D$ is nearly cloaked when $\rho$ is small. More precisely, when the conductivity of $\Omega$ has the form

$$
\sigma_{A}(y)= \begin{cases}A(y) & \text { for } y \in D \\ H_{*} 1(y) & \text { for } y \in \Omega \backslash D,\end{cases}
$$

the Dirichlet-to-Neumann map is nearly independent of $A$ in the sense that

$$
\left\|\Lambda_{\sigma_{A}}-\Lambda_{1}\right\| \leqslant C \rho^{n}
$$

\section{Analysis of the singular cloak}

This section discusses the perfect cloak obtained using the singular change of variables (12). We focus on the radial case for simplicity, but our argument extends straightforwardly to a broad class of non-radial examples (see theorem 4).

As we explained in section 2.3, the basic assertion of cloaking is that for conductivities of the form (11) with $F$ given by (12), the Dirichlet-to-Neumann map is identical to that of the uniform ball with conductivity 1 . Thus, if the shell $B_{2} \backslash B_{1}$ has conductivity $F_{*} 1$ then the ball $B_{1}$ is cloaked. 
This assertion follows from theorem 1 by passing to the limit $\rho \rightarrow 0$ (see remark 1 in section 4.2). But it can also be proved directly, and the direct argument-being very different_-gives additional insight. In particular, it reveals the mechanism of cloaking: the potential in $B_{1}$ is constant, rendering the conductivity in this region irrelevant.

The essence of the argument presented in this section is similar to that of [19]. In particular, our main tool is a well-known result on the removability of isolated singularities for solutions of Laplace's equation (see the proof of proposition 2).

\subsection{Explicit form of the cloak}

Recall that $F_{*} 1$ is defined by (8). When $F: B_{2} \rightarrow B_{2}$ is given by (12) it is easy to make $F_{*} 1$ explicit. Indeed, the Jacobian matrix $D F=\left(\partial F_{i} / \partial x_{j}\right)$ is

$$
D F=\left(\frac{1}{2}+\frac{1}{|x|}\right) I-\frac{1}{|x|} \hat{x} \hat{x}^{T},
$$

for $x \neq 0$, where $I$ is the identity matrix and $\hat{x}=x /|x|$. Thus $D F$ is symmetric, $\hat{x}$ is an eigenvector with eigenvalue $1 / 2$ and (in space dimension $n) \hat{x}^{\perp}$ is an $(n-1)$-dimensional eigenspace with eigenvalue $\frac{1}{2}+\frac{1}{|x|}$. The determinant is evidently

$$
\operatorname{det}(D F)=\frac{1}{2}\left(\frac{1}{2}+\frac{1}{|x|}\right)^{n-1}=\frac{(|x|+2)^{n-1}}{2^{n}|x|^{n-1}}
$$

It follows by a brief calculation that in the shell $1<|y|<2$,

$$
F_{*} 1(y)=\frac{2^{n}}{(2+|x|)^{n-1}}\left[\left(\frac{1}{4}|x|^{n-1}+|x|^{n-2}+|x|^{n-3}\right)\left(I-\hat{x} \hat{x}^{T}\right)+\frac{1}{4}|x|^{n-1} \hat{x} \hat{x}^{T}\right],
$$

where the right-hand side is evaluated at

$$
x=F^{-1}(y)=2(|y|-1) \frac{y}{|y|} .
$$

Since $F$ is singular at $x=0$ we expect $F_{*} 1$ to be a bit strange near the inner boundary of the shell. The details depend on the spatial dimension $n$ :

$$
\begin{aligned}
& \text { when } n=2 \text {, one eigenvalue of } F_{*} 1 \text { tends to } 0 \text { and the other to } \infty \text {; } \\
& \text { when } n=3 \text {, one eigenvalue tends to } 0 \text { while the others remain finite; } \\
& \text { when } n \geqslant 4 \text {, all eigenvalues tend to } 0 \text {. }
\end{aligned}
$$

In fact: writing $r=|x|=2(|y|-1)$, when $n=2$ the eigenvalues behave like $r$ and $r^{-1}$ as $r \rightarrow 0$; when $n=3$ one eigenvalue behaves like $r^{2}$ and two like $r^{0}$; when $n \geqslant 4$ one eigenvalue behaves like $r^{n-1}$ and the remaining $n-1$ like $r^{n-3}$. Note that for $n \geqslant 3$, the conductivity $F_{*} 1$ depends smoothly on $y$ near the inner boundary of the shell. The 'strangeness' we mentioned above is not a lack of smoothness but rather a degeneracy (lack of a uniform lower bound). In space dimension $n=2$ the situation is little different: $F_{*} 1$ becomes degenerate but also lacks smoothness since the circumferential eigenvalue becomes infinite. This difference between $n=2$ and $n \geqslant 3$ will play no essential role in our analysis.

\subsection{The potential outside the cloaked region}

Let $v$ be the potential associated with Dirichlet data $f$ :

$$
\nabla \cdot\left(\sigma_{A} \nabla v\right)=0 \quad \text { in } \quad B_{2}, \quad \text { with } \quad v=f \quad \text { at } \partial B_{2},
$$


where $\sigma_{A}$ is given by (11) using the singular change of variable (12). We assume, as in section 3, that $A$ is bounded above and below in the sense that (22) holds.

Does this PDE have a unique solution? The answer is not immediately obvious, due to the degeneracy of $F_{*} 1$ near $|y|=1$. We shall show, here and in section 4.3 , that the only reasonable solution of $(31)$ is

$$
v(y)= \begin{cases}u(x) & \text { for } y \in B_{2} \backslash B_{1} \\ u(0) & \text { for } y \in B_{1},\end{cases}
$$

where $u$ is the harmonic function with the same Dirichlet data

$$
\Delta u=0 \quad \text { in } \quad B_{2}, \quad \text { with } \quad u=f \quad \text { at } \quad \partial B_{2}
$$

and $x=F^{-1}(y)$.

What can we assume about the solution of (31)? Later, in section 4.3 , we will ask that $\nabla v$ and $\sigma_{A} \nabla v$ both be square-integrable. For the moment, however, we ask only that $v$ be bounded near $|y|=1$. More precisely, we ask that

$$
|v(y)| \leqslant C \quad \text { for } \quad|y| \leqslant r
$$

for some constants $C<\infty$ and $1<r<2$. (We do not assume $v$ is bounded in the entire ball $B_{2}$ because the Dirichlet data can be unbounded-an $H^{1 / 2}$ function need not be $L^{\infty}$.) This is a very modest hypothesis. Indeed, since $F_{*} 1$ is smooth for $|y|>1$, elliptic regularity assures us that $v$ is uniformly bounded in any compact subset of $B_{2} \backslash B_{1}$. The essential content of (34) is thus that $v$ does not diverge as $|y| \rightarrow 1$. If the conductivity were positive and finite such growth would be ruled out by the variational principle (5) and an easy truncation argument.

With this modest hypothesis on $v$, we can identify its values in $B_{2} \backslash B_{1}$ by changing variables then using a standard theorem about the removability of point singularities for harmonic functions.

Proposition 2. If $v$ solves (31) and satisfies (34) then

$$
v(y)=u(x) \quad \text { for } 1<|y|<2,
$$

where $x=F^{-1}(y)$ and $u$ is the harmonic function on $B_{2}$ with the same Dirichlet data as $v$.

Proof. Since $\sigma_{A}(y)=F_{*} 1(y)$ is smooth and bounded away from zero for $|y|$ strictly larger than 1, elliptic regularity applies and $v$ is a classical solution of the PDE in $B_{2} \backslash \overline{B_{1}}$. When $\phi$ is supported in $B_{2} \backslash \overline{B_{1}}$, the PDE combines with the definition of $F_{*}$ and the change-of-variables formula to give

$$
0=\int\left\langle\sigma_{A} \nabla_{y} v(y), \nabla_{y} \phi(y)\right\rangle \mathrm{d} y=\int\left\langle\nabla_{x} v(F(x)), \nabla_{x} \phi(F(x)\rangle \mathrm{d} x .\right.
$$

Since $\phi(y)$ is supported on $B_{2} \backslash \overline{B_{1}}$, the test function $\phi(F(x))$ vanishes at 0 and $\partial B_{2}$ but is otherwise arbitrary. So (36) tells us that $w(x)=v(F(x))$ is a weak solution of $\Delta w=0$ in the punctured ball $B_{2} \backslash\{0\}$. By elliptic regularity, it is also a classical solution.

We now use the following well-known result about removable singularities for harmonic functions: if $\Delta w=0$ in a punctured ball about 0 and if

$$
\begin{array}{ll}
|w(x)|=o\left(|x|^{2-n}\right) & \text { in dimension } n \geqslant 3, \text { or } \\
|w(x)|=o\left(\log |x|^{-1}\right) & \text { in dimension } n=2
\end{array}
$$

as $x \rightarrow 0$, then $w$ has a removable singularity at 0 (see e.g. [15]). In other words, $w(0)$ is determined by continuity and (so extended) $w$ is harmonic in the entire ball.

Our $w(x)=v(F(x))$ satisfies (37) - indeed, it is uniformly bounded near 0 as a consequence of (34). So $w$ is harmonic on $B_{2}$. Moreover $w$ has the same Dirichlet data 
as $v$, since $F(x)=x$ at $\partial B_{2}$. Thus $w$ is precisely the function $u$ that appears in (35) and the proof is complete.

Remark 1. We have shown using elliptic theory that for the cloak constructed using the singular change of variable (12), the potential outside the cloaked region is given by (35). An alternative, more physical justification of (35) is this: it gives the limiting value of the potential associated with our regular near-cloak (10) in the singular limit $\rho \rightarrow 0$.

To justify the remark, let $F_{\rho}$ be the regularized change of variable (10), and let $v_{\rho}$ be the potential in the near-cloak for a given choice of the Dirichlet data. Then $u_{\rho}(x)=v_{\rho}\left(F_{\rho}(x)\right)$ is harmonic outside $B_{\rho}$. It is also uniformly bounded (away from the outer boundary $|y|=2$ ), with a bound independent of $\rho$. So by a standard compactness argument, the limit as $\rho \rightarrow 0$ exists and is harmonic in $B_{2} \backslash\{0\}$. Since the limit is bounded, 0 is a removable singularity and $u_{0}(x)=\lim _{\rho \rightarrow 0} u_{\rho}(x)$ is the unique harmonic function in $B_{2}$ with the given Dirichlet data. Now for any fixed $1<|y|<2$ we can pass to the limit $\rho \rightarrow 0$ in the relation $v_{\rho}(y)=u_{\rho}\left(F_{\rho}^{-1}(y)\right)$ to get $v_{0}(y)=u_{0}\left(F_{0}^{-1}(y)\right)$, confirming (35).

\subsection{The potential inside the cloaked region}

We have asserted that the solution of (31) is given by (32). Proposition 2 justifies this assertion outside $B_{1}$; this section completes the justification by showing that (i) the proposed $v$ is indeed a solution and (ii) it is the only reasonable solution.

To show that $v$ is a solution, we must demonstrate that $\sigma_{A} \nabla v$ is divergence-free. This is the main goal of the following proposition.

Proposition 3. Fixing $f \in H_{*}^{1 / 2}\left(\partial B_{2}\right)$, let $v$ be defined by (32). Then

(a) $v$ is Lipschitz continuous away from $\partial B_{2}$, i.e. $|\nabla v|$ is uniformly bounded in $B_{r}$ for every $r<2$;

(b) $\sigma_{A} \nabla v$ is also uniformly bounded away from $\partial B_{2}$;

(c) $\left(\sigma_{A} \nabla v\right) \cdot v \rightarrow 0$ uniformly as $|y| \downarrow 1$, where $v=y /|y|$ is the normal to $\partial B_{1}$;

(d) $\sigma_{A} \nabla v$ is weakly divergence-free in the entire domain $B_{2}$.

Proof. We observe first that (d) follows immediately from (b), (c) and (36). Indeed, a bounded vector-field $\xi$ is weakly divergence-free on $B_{2}$ if and only if it is weakly divergence-free on the subdomains $B_{1}$ and $B_{2} \backslash \overline{B_{1}}$ and its normal flux $\xi \cdot v$ is continuous across the interface $\partial B_{1}$. (The normal flux is well defined from either side, as a consequence of $\xi$ being divergence free in $B_{1}$ and its complement.) We apply this to $\xi=\sigma_{A} \nabla v$, which is clearly divergence-free in $B_{1}$ (where it vanishes) and in $B_{2} \backslash \overline{B_{1}}$ (by equation (36)). If (c) holds then the normal flux $\xi \cdot v=0$ vanishes on both sides of $\partial B_{1}$. In particular it is continuous, so (d) holds.

The proofs of (a)-(c) are straightforward calculations based on the change-of-variables formula and the smoothness of $u(x)=v(F(x))$, together with our explicit formulae for $D F$ (24) and $F_{*} 1$ (26). To see that $\nabla v$ is bounded away from $\partial B_{2}$ we observe that, by chain rule and the symmetry of $D F$, we have

$$
\nabla_{y} v=\left(D F^{-1}\right)^{T} \nabla_{x} u=(D F)^{-1} \nabla_{x} u
$$

for $1<|y|<2$. The matrix $(D F)^{-1}$ is uniformly bounded, by (24); and $\nabla_{x} u$ is bounded (except perhaps near $\partial B_{2}$ ) since $u$ is harmonic in $x$. Thus $|\nabla v|$ is bounded and $v$ is Lipschitz continuous on $1 \leqslant|y|<r$ for any $r<2$. It is moreover constant on $B_{1}$, and continuous across $\partial B_{1}$. Therefore $v$ is Lipschitz continuous on the entire ball $B_{r}$ for every $r<2$. 
In dimensions $n \geqslant 3$ (b) follows immediately from (a), since $F_{*} 1$ is uniformly bounded. In dimension $n=2$ however we must be more careful, since $F_{*} 1$ becomes unbounded as $|y| \downarrow 1$. Using the definition of $\sigma_{A}$, chain rule and the symmetry of $D F$ we have

$$
\sigma_{A} \nabla_{y} v=F_{*} 1(D F)^{-1} \nabla_{x} u
$$

for $1<|y|<2$. The symmetric matrices $F_{*} 1$ and $(D F)^{-1}$ have the same eigenvectors, namely $\hat{x}$ and $\hat{x}^{\perp}$. Taking $n=2$ in (24) and (26) we see that the eigenvalue of $F_{*} 1$ in direction $\hat{x}^{\perp}$ behaves like $|x|^{-1}$, while that of $(D F)^{-1}$ behaves like $|x|$. The eigenvalues of both matrices in direction $\hat{x}$ are bounded. Thus the product $F_{*} 1(D F)^{-1}$ is bounded. This yields (b), since $\nabla_{x} u$ is bounded away from $\partial B_{2}$ and $\sigma_{A} \nabla v=0$ for $y \in B_{1}$.

The proof of (c) is similar to that of (b). Since $|y| \downarrow 1$ corresponds to $|x| \rightarrow 0$ and $y /|y|=x /|x|=\hat{x}$, we must show that the $\hat{x}$ component of (38) tends to zero as $|x| \rightarrow 0$. Since $F_{*} 1(D F)^{-1}$ is symmetric and $\hat{x}$ is an eigenvector, it suffices to show that the corresponding eigenvalue tends to 0 . In fact, its value according to (24) and (26) is

$$
\frac{2^{n-1}}{(2+|x|)^{n-1}}|x|^{n-1} \leqslant|x|^{n-1}
$$

which tends to zero linearly (if $n=2$ ) or better (if $n \geqslant 3$ ). The proof is now complete.

We have shown that the function defined by (32) solves the PDE (31). Is it the only solution? If $\sigma_{A}$ were strictly positive and finite, uniqueness would be standard. When $\sigma_{A}$ is degenerate, however, uniqueness can sometimes fail. For example, if $\sigma_{A}$ were identically 0 in $B_{1}$ then the solution would not be unique: $v$ would be arbitrary in $B_{1}$. Our situation, however, is much more controlled: the degeneracy occurs only at $\partial B_{1}$, and it has a very specific form.

Uniqueness should be proved in a specific class. We assumed in section 4.2 that $v$ was uniformly bounded near $\partial B_{1}$. Here we assume further that

$$
\nabla v \in L^{2}\left(B_{2}\right) \quad \text { and } \quad \sigma_{A} \nabla v \in L^{2}\left(B_{2}\right) .
$$

Proposition 4. If $v$ is a weak solution of the PDE (31) which also satisfies (34) and (39) then $v$ must be given by formula (32).

Proof. We know from proposition 2 that $v(y)=u(x)$ outside $B_{1}$. What remains to be proved is that $v \equiv u(0)$ in $B_{1}$.

Recall that $u$ has a removable singularity at 0 . In particular it is continuous there. Since $F^{-1}$ maps $\partial B_{1}$ to 0 , it follows that $v(y) \rightarrow u(0)$ as $y$ approaches $\partial B_{1}$ from outside.

Since $\nabla v \in L^{2}\left(B_{2}\right)$ by hypothesis, the restriction of $v$ to $\partial B_{1}$ makes sense, and it is the same from outside or inside. Evidently this restriction is constant, identically equal to $u(0)$. It follows, by uniqueness for the $\operatorname{PDE} \nabla \cdot(A \nabla v)=0$ in $B_{1}$, that $v \equiv u(0)$ throughout $B_{1}$, as asserted.

The preceding argument actually uses somewhat less than (39). Any condition that makes $v$ continuous across $\partial B_{1}$ would be sufficient. However we also need a hypothesis on $\sigma_{A} \nabla v$ (for example that it be integrable) for the PDE (31) to make sense.

\subsection{The singular cloak is invisible}

Our main point is that if the shell $B_{2} \backslash B_{1}$ has conductivity $F_{*} 1$ then the ball $B_{1}$ is cloaked. This is an easy consequence of the preceding results: 
Theorem 3. Suppose $\sigma_{A}$ is given by (11), where $F$ is given by (12) and $A$ is uniformly positive and finite (22). Then the associated Dirichlet-to-Neumann map $\Lambda_{\sigma_{A}}$ is the same as that of a uniform ball $B_{2}$ with conductivity 1.

Proof. It suffices to prove that $\Lambda_{\sigma_{A}}$ and $\Lambda_{1}$ determine the same quadratic form on Dirichlet data, where $\Lambda_{1}$ is the Dirichlet-to-Neumann map of the uniform ball. But by (32) we have

$$
\int_{B_{2}}\left\langle\sigma_{A} \nabla v, \nabla v\right\rangle \mathrm{d} y=\int_{B_{2} \backslash B_{1}}\left\langle\sigma_{A} \nabla v, \nabla v\right\rangle \mathrm{d} y
$$

and the definition of $\sigma_{A}$ combined with the change-of-variables formula gives

$$
\int_{B_{2} \backslash B_{1}}\left\langle\sigma_{A} \nabla v \nabla v\right\rangle \mathrm{d} y=\int_{B_{2}}\left|\nabla_{x} u\right|^{2} \mathrm{~d} x
$$

where $u$ is harmonic with the same Dirichlet data as $v$. Thus

$$
\left\langle\Lambda_{\sigma_{A}} f, f\right\rangle=\left\langle\Lambda_{1} f, f\right\rangle
$$

for all $f \in H_{*}^{1 / 2}$, where $\Lambda_{\sigma_{A}}=\Lambda_{1}$ as asserted.

We have focused on the radial setting for the sake of simplicity. However the analysis in this section extends straightforwardly to the nonradial cloaks discussed at the end of section 3 .

Theorem 4. Let $G: B_{2} \rightarrow \Omega$ be a Lipschitz continuous map with Lipschitz continuous inverse, and let $D=G\left(B_{1}\right)$. Then $H=G \circ F \circ G^{-1}: \Omega \rightarrow \Omega$ acts as the identity on $\partial \Omega$, while 'blowing up' the point $z_{0}=G(0)$ to $D$. (This is the $\rho=0$ limit of figure 3 ). Consider a conductivity $\sigma_{A}$ defined on $\Omega$ of the form

$$
\sigma_{A}(w)= \begin{cases}A(w) & \text { for } w \in D \\ H_{*} 1(w) & \text { for } w \in \Omega \backslash D,\end{cases}
$$

where $A$ is symmetric, positive and finite but otherwise arbitrary. The associated Dirichletto-Neumann map $\Lambda_{\sigma_{A}}$ is independent of $A$; in fact, $\Lambda_{\sigma_{A}}=\Lambda_{1}$ is the Dirichlet-to-Neumann map associated with conductivity 1.

Proof. We claim that

$$
v(w)= \begin{cases}u(z) & \text { for } w \in \Omega \backslash D \\ u\left(z_{0}\right) & \text { for } w \in D,\end{cases}
$$

where $w=H(z)$ and $u$ solves $\Delta u=0$ in $\Omega$ with the same Dirichlet data as $v$. The proof is parallel to our argument in the radial case, so we shall be relatively brief.

The proof of proposition 2 made no use of radial symmetry; it applies equally in the present setting. We must assume of course that $v$ is bounded away from $\partial \Omega$, and we conclude that (40) is correct outside $D$.

The analogue of proposition 3(a) is the statement that $v$ is uniformly Lipschitz in $\Omega \backslash \bar{D}$ except perhaps near $\partial \Omega$. With the conventions $x=G^{-1}(z), y=F(x)$ and $w=G(y)$, we have

$$
D H(z)=D G(y) D F(x) D G^{-1}(z)
$$

by chain rule. By hypothesis, $D G$ and $D G^{-1}$ are uniformly bounded. Therefore $(D H)^{-1}$ is uniformly bounded too. Since $\Delta_{z} u=0, u$ is a smooth function of $z$ except perhaps near $\partial \Omega$. It follows that $v(w)=u\left(H^{-1}(w)\right)$ is uniformly Lipschitz continuous away from $\partial \Omega$. 
The analogue of proposition $3(\mathrm{~b})$ is the statement that $H_{*} 1 \nabla_{w} v$ is uniformly bounded away from $\partial \Omega$. Recalling the definition

$$
H_{*} 1=\frac{1}{\operatorname{det} D H} D H D H^{T}
$$

and using that $\nabla_{w} v=\left(D H^{T}\right)^{-1} \nabla_{z} u$, we see that

$$
H_{*} 1 \nabla_{w} v=\frac{1}{\operatorname{det} D H} D H \nabla_{z} u
$$

Since $u$ is harmonic, it is smooth away from $\partial \Omega$. As for $D H / \operatorname{det}(D H)$ : it has the same behaviour as $D F / \operatorname{det}(D F)$, since $D G$ and $D G^{-1}$ are bounded. One verifies using the explicit formula (24) that $D F / \operatorname{det}(D F)$ stays bounded as $x \rightarrow 0$.

The analogue of proposition 3(b) is the statement that the normal flux $\left(H_{*} 1 \nabla_{w} v\right) \cdot n_{w} \rightarrow 0$ as $w$ approaches $\partial D$ from outside, where $n_{w}$ is the unit normal at $\partial D$. We use the fact that $n_{w}$ is parallel to $\left(D G^{-1}\right)^{T}\left(v_{y}\right)$, if $v_{y}$ is the unit normal to $\partial B_{1}$ at the corresponding point $y=G^{-1}(w)$. (To see this, note that if $\tau$ is tangent to $\partial B_{1}$ then $D G \tau$ is tangent to $\partial D$, and $\left\langle D G \tau,\left(D G^{-1}\right)^{T} \nu\right\rangle=\langle\tau, v\rangle=0$.) It follows that

$$
\left|\left(H_{*} 1 \nabla_{w} v\right) \cdot n_{w}\right| \leqslant C\left|\left\langle H_{*} 1 \nabla_{w} v,\left(D G^{-1}\right)^{T} v_{y}\right\rangle\right| .
$$

Now,

$$
H_{*} 1 \nabla_{w} v=(\operatorname{det} D H)^{-1} D H \nabla_{z} u=(\operatorname{det} D H)^{-1} D G D F D G^{-1} \nabla_{z} u .
$$

So the inner product on the right-hand side of (41) is equal to $(\operatorname{det} D H)^{-1}\left|\left\langle D G D F D G^{-1} \nabla_{z} u,\left(D G^{-1}\right)^{T} v_{y}\right\rangle\right|=(\operatorname{det} D H)^{-1}\left|\left\langle D F D G^{-1} \nabla_{z} u, v_{y}\right\rangle\right|$.

Since $D G$ and $D G^{-1}$ are bounded, this is bounded by a constant times

$$
(\operatorname{det} D F)^{-1}\left|\left\langle D F D G^{-1} \nabla_{z} u, v_{y}\right\rangle\right| \text {. }
$$

But recall that $v_{y}=y /|y|=x /|x|$ is an eigenvector of the symmetric matrix $(\operatorname{det} D F)^{-1} D F$, with an eigenvalue that tends to 0 as $x \rightarrow 0$. Therefore

$$
\left(H_{*} 1 \nabla_{w} v\right) \cdot n_{w} \rightarrow 0 \quad \text { as } \quad w \rightarrow D,
$$

as asserted.

The arguments used for proposition 3(d), proposition 4 and theorem 3 did not use radial symmetry or the explicit form of the cloak, so they extend immediately to the present setting.

We note that for $n \geqslant 3$ the results in theorems 3 and 4 coincide with those already established in [19].

\section{Acknowledgments}

This work was supported by NSF through grants DMS-0313744 and DMS-0313890 (RVK and HS), DMS-0412305 and DMS-0707850 (MIW), and DMS-0604999 (MSV). We thank the anonymous referee for his constructive criticism, which significantly improved the paper.

\section{References}

[1] Adams R A 1975 Sobolev Spaces (New York: Academic)

[2] Ammari H and Kang H 2004 Reconstruction of Small Inhomogeneities from Boundary Measurements (Lecture Notes in Mathematics vol 1846) (Berlin: Springer) 
[3] Ammari H, Vogelius M S and Volkov D 2001 Asymptotic formulae for perturbations in the electromagnetic fields due to the presence of inhomogeneities of small diameter: II. The full Maxwell equations J. Math. Pures Appl. 80 769-814

[4] Astala K and Päivärinta L 2006 Calderón's inverse conductivity problem in the plane Ann. Math. 163 265-99

[5] Astala K, Päivärinta L and Lassas M 2005 Calderón's inverse problem for anisotropic conductivity in the plane Commun. PDE 30 207-24

[6] Cai W, Chettiar U K, Kildishev A V and Shalaev V M 2007 Optical cloaking with metamaterials Nature Photon. $1224-7$

[7] Calderón A P 1980 On an inverse boundary value problem Seminar on Numerical Analysis and its Applications to Continuum Physics (Rio de Janeiro: Soc. Brasileira de Matemática) pp 65-73

[8] Chang K 2007 Flirting with invisibility, New York Times, Science Times

[9] Chen H and Chan C T 2007 Transformation media that rotate electromagnetic fields Appl. Phys. Lett. 90241105

[10] Chen H, Wu B-I, Zhang B and Kong J A 2007 Electromagnetic wave interactions with a metamaterial cloak Phys. Rev. Lett. 99063903

[11] Cheney M, Isaacson D and Newell J C 1999 Electrical impedance tomography SIAM Rev. 41 85-101

[12] Cummer S A, Popa B-I, Schurig D and Smith D R 2006 Full-wave simulations of electromagnetic cloaking structures Phys. Rev. E 74036621

[13] Cummer S A and Schurig D 2007 One path to acoustic cloaking New J. Phys. 945

[14] Druskin V L 1985 On uniqueness of the determination of three-dimensional underground structures from surface measurements with variously positioned steady-state or monochromatic field sources Sov. Phys.-Solid Earth 21 210-4 (Engl. trans. Washington, DC: American Geophysical Union)

[15] Folland G B 1976 Introduction to Partial Differential Equations (Princeton, NJ: Princeton University Press)

[16] Friedman A and Vogelius M 1989 Identification of small inhomogeneities of extreme conductivity by boundary measurements: a theorem on continuous dependence Arch. Ration. Mech. Anal. 105 299-326

[17] Greenleaf A, Kurylev Y, Lassas M and Uhlmann G 2007 Full-wave invisibility of active devices at all frequences Commun. Math. Phys. 275 749-89

[18] Greenleaf A, Kurylev Y, Lassas M and Uhlmann G 2007 Improvement of cylindrical cloaking with the SHS lining Opt. Exp. 15 12717-34

[19] Greenleaf A, Lassas M and Uhlmann G 2003 On nonuniqueness for Calderon's inverse problem Math. Res. Lett. 10 685-93

[20] Greenleaf A, Lassas M and Uhlmann G 2003 Anisotropic conductivities that cannot be detected by EIT Physiol. Meas. 24 413-9

[21] Isakov V 1997 Inverse Problems for Partial Differential Equations (Berlin: Springer)

[22] Kohn R V and Vogelius M 1984 Identification of an unknown conductivity by means of measurements at the boundary Inverse Problems ed D W McLaughlin (SIAM-AMS Proc. vol 14) (Providence, RI: American Mathematical Society) pp 113-23

[23] Kohn R V and Vogelius M 1984 Determining conductivity by boundary measurements Commun. Pure Appl. Math. 37 289-98

[24] Kohn R V and Vogelius M 1985 Determining conductivity by boundary measurements: II. Interior results Commun. Pure Appl. Math. 38 643-67

[25] Kohn R V and Vogelius M 1987 Relaxation of a variational method for impedance computed tomography Commun. Pure Appl. Math. $\mathbf{4 0} 745-77$

[26] Lassas M 1997 The impedance imaging problem as a low-frequency limit Inverse Problems 13 1503-18

[27] Lee J and Uhlmann G 1989 Determining anisotropic real-analytic conductivities by boundary measurements Commun. Pure Appl. Math. 42 1097-112

[28] Leonhardt U 2006 Optical conformal mapping Science 312 1777-80

[29] Leonhardt U 2006 Notes on conformal invisibility devices New J. Phys. 8118

[30] Miller D A B 2006 On perfect cloaking Opt. Exp. 14 12457-66

[31] Milton G, Briane M and Willis J R 2006 On cloaking for elasticity and physical equations with a transformation invariant form New J. Phys. 8248

[32] Milton G W and Nicorovici N-A P 2006 On the cloaking effects associated with anomalous localized resonance Proc. R. Soc. A 462 3027-59

[33] Nachman A I 1996 Global uniqueness for a two-dimensional inverse boundary value problem Ann. Math. $14371-96$

[34] Ola P, Päivärinta L and Somersalo E 1993 An inverse boundary value problem in electrodynamics Duke Math. J. 70 617-53

[35] Pendry J B, Schurig D and Smith D R 2006 Controlling electromagnetic fields Science 312 1780-2 
[36] Ramm A 1996 Minimization of the total radiation from an obstacle by a control function on a part of its boundary J. Inverse Ill-Posed Problems 4 531-4

[37] Ruan Z, Yan M, Neff C W and Qiu M 2007 Ideal cylindrical cloak: perfect but sensitive to tiny perturbations Phys. Rev. Lett. 99113903

[38] Schurig D, Pendry J B and Smith D R 2006 Calculation of material properties and ray tracing in transformation media Opt. Exp. 14 9794-804

[39] Schurig D, Mock J J, Justice B J, Cummer S A, Pendry J B, Starr A F and Smith D R 2006 Metamaterial electromagnetic cloak at microwave frequencies Science $\mathbf{3 1 4} 977-80$

[40] Smith D R, Pendry J B and Wiltshire M C K 2004 Metamaterials and negative refractive index Science $305788-92$

[41] Sylvester J 1990 An anisotropic inverse boundary value problem Commun. Pure Appl. Math. 43 201-32

[42] Sylvester J and Uhlmann G 1987 A global uniqueness theorem for an inverse boundary value problem Ann. Math. 125 153-69

[43] Uhlmann G 1999 Developments in inverse problems since Calderón's foundational paper Harmonic Analysis and Partial Differential Equations: Chicago Lectures in Math. (Chicago, IL, 1996) (Chicago, IL: University of Chicago Press) pp 295-345

[44] Borcea L 2002 Electrical Impedance Tomography Inverse Problems 18 R99-136

[45] Wilson M 2007 Designer materials render objects nearly invisible to microwaves Phys. Today 60 19-23

[46] Zolla F, Guenneau S, Nicolet A and Pendry J B 2007 Electromagnetic analysis of cylindrical invisibility cloaks and the mirage effect Opt. Lett. 32 1069-71 